\title{
Inklusive Bildung und Fachdidaktik in Grundschulen. Erkenntnisse, Reflektionen und Perspektiven
}

\author{
Simone Seitz • Toni Simon
}

Eingegangen: 9. Juni 2020 / Angenommen: 27. Juli 2020 / Online publiziert: 18. Januar 2021

(C) Der/die Autor(en) 2021

Zusammenfassung Ausgehend von der Frage, inwiefern die Grundschule ihrem eigenen Anspruch gemäß ein Ort allgemeiner Bildung für alle Kinder ist, wird ein Überblick über Theorien und Modelle inklusiver (grund)schulischer Bildung und deren Entwicklung von der frühen Integrationsforschung bis zur aktuellen Inklusionsforschung mit Fokus auf fach- bzw. lernbereichsdidaktische Fragestellungen in Grundschulen gegeben. Auf dieser Basis wird exemplarisch der Sachunterricht, dem im Hinblick auf die Lernbereiche der Grundschule eine exponierte Position zukommt, genauer beleuchtet, bevor dann abschließend Perspektiven für die zukünftige grundschulpädagogische Forschung im Feld inklusiver Bildung und inklusionsbezogener Fachdidaktiken abgeleitet werden.

Schlüsselwörter Inklusive Bildung · Fachdidaktik · Allgemeinbildung · Sachunterricht

Prof. Dr. S. Seitz $(\varangle)$

Professur für Allgemeine Didaktik mit Schwerpunkt Inklusion, Fakultät für Bildungswissenschaften, Freie Universität Bozen - Libera Università di Bolzano, Regensburger Allee 16 Viale Ratisbona, 39042 Brixen Bressanone, Italien

E-Mail: simone.seitz@unibz.it

Dr. T. Simon

Philosophische Fakultät III - Erziehungswissenschaften, Institut für Schulpädagogik und Grundschuldidaktik, Martin-Luther-Universität Halle-Wittenberg, Franckeplatz 1, 06110 Halle, Deutschland

E-Mail: toni.simon@paedagogik.uni-halle.de 


\title{
Inclusive education and subject-specific learning in primary schools. Findings, reflections and perspectives
}

\begin{abstract}
Based on the question how far primary schools are a place of general education for all children according to their own aspirations, the article gives an overview of theories and models of inclusive (primary) school education and their development from early integration research to current inclusion research in the light of subject-related or learning area-related didactics in primary schools. On this basis, general studies, which is in an exposed position with regard to the learning areas of primary school, will be examined in more detail, before perspectives for future research in the field of inclusive education and inclusive subject didactics will be derived.
\end{abstract}

Keywords Inclusive education - Subject-related teaching and learning - General education $\cdot$ Social studies and science learning

\section{Die Grundschule als Ort allgemeiner Grundbildung für alle Kinder}

Die Grundschule wurde 1919 als institutioneller Ort grundlegender Bildung für alle Kinder gegründet. Mit der auch damals schon umstrittenen Dauer von (nur) vier gemeinsamen Schuljahren wurde in Abkehr von der Standesschule der Gedanke verknüpft, allen Kindern unterschiedslos den Zugang zu grundlegender Bildung zu eröffnen. Zugleich wurde der Grundschule eine integrative Funktion zugeordnet (Einsiedler 2005), womit die pädagogische Dimension innerhalb dieser Schulstufe eine zentrale Position erhielt. Sie ist jedoch im deutschen Bildungssystem bis in die Gegenwart mit dem prognostisch angelegten Zuweisungsauftrag für die Sekundarstufe in institutionelle Dynamiken der Selektion eingebunden, die ihre Rolle mit Ambivalenzen ausstatten (Diehm 2020). In der Grundschule finden sich somit die widersprüchlichen institutionengebundenen Aufträge der Schule insgesamt in zugespitzter Form wieder - was auch als strukturelle Einschreibung professionsbedingter Antinomien des Handelns von Lehrpersonen (Helsper 2014) verstanden werden kann. Ihr kommt somit eine Schlüsselposition im Bildungssystem wie zugleich in individuellen Bildungsbiografien zu, während die ihr obliegende Aufgabe der Grundbildung in Deutschland von institutionell widerspruchsreichen und dichten Anforderungen gekennzeichnet ist.

Seit langem wird in grundschulpädagogischen Diskursen unter regelmäßigem Bezug auf Klafkis theoretische Grundlegungen der Allgemeinbildung diskutiert, dass sich kindliches Lernen fächerstruktureller Verengungen enthebt - was Klafki selbst im Konzept des fächerübergreifenden Unterrichts an epochaltypischen Schlüsselproblemen fasste, ein Ansatz der zudem zentral der Idee einer demokratischen und humanen Schule verpflichtet ist (Klafki 1994). Bereits 1996 wurde dies in der Schrift „Zukunft der Grundschule“ (Faust-Siehl et al. 1996) eindrucksvoll aufgenommen. „Bildend im engeren Sinne“ so heißt es dort, ,sind jedoch weder die Wissenschaftsdisziplinen noch die aus ihnen abgeleiteten Schulfächer, sondern nur die Auseinandersetzung mit kulturell bedeutsamen Fragestellungen, die notwendigerweise die 
Grenzen von Disziplinen und Fächern sprengen“ (ebd., S. 58). Hier anknüpfend werden Lernbereiche als übergreifende Klammer verstanden und die Grundschule insgesamt als Ort des projektorientierten und fächerübergreifenden Lernens an Schlüsselproblemen konzipiert (ebd., S. 73). Strukturell ist jedoch in Deutschland allein der Sachunterricht als fächerintegrierender Lernbereich fest installiert worden und das Verhältnis von Allgemeiner Didaktik und Fachdidaktik in Forschung und Lehrer*innenbildung ist auch primarstufenbezogen weiterhin Gegenstand lebendig geführter Fachdiskurse (Arnold et al. 2010).

Wie in keinem anderen Lernbereich wurde im Sachunterricht das bildungstheoretische Konzept der Allgemeinbildung sensu Klafki in eine integrierende fachliche Struktur überführt, die Mehrperspektivität substantiell aufgenommen und konzeptionell ausbuchstabiert hat (u. a. Richter 2002; Kaiser und Pech 2004). Auch in den vor allem in der Primarstufe diskutierten und hier besonders weit entwickelten Konzepten geöffneten bzw. offenen Unterrichts, die ebenfalls unter der Zielebene einer stärkeren Demokratisierung der Schule entwickelt wurden, finden wir das kindorientierte, interessengeleitete Lernen unter der Leitidee der allgemeinen Grundbildung im Zentrum, von wo aus fachdidaktische Lernprozesse entworfen werden - nicht umgekehrt (u. a. Peschel 2006a, S. 37, 2006b).

Die Grundschule ist damit wie keine andere Bildungsstufe in der Lage, die Ansprüche demokratischer, allgemeiner Grundbildung für alle Kinder zu erfüllen, steht jedoch zugleich wie oben angedeutet in struktur-funktionalen Widersprüchen, die nicht folgenlos sind für die Handlungspraxis und zu Diskrepanzen führen zwischen dem Anspruch, eine „Schule für alle Kinder“ zu sein und den konkreten Handlungsmöglichkeiten (Seitz 2014; Miller et al. 2019; Simon 2020). So ist sie zweifellos hinsichtlich der Verhandlung von Heterogenität in Unterricht und Schulleben die konzeptionell am weitesten entwickelte Schulform im deutschen Bildungssystem, umspannt jedoch innerhalb des weiterhin primär an Homogenität ausgerichteten Schulsystems die kürzeste Phase von Bildungsbiografien und weist auch für sich genommen noch immer einzelne (leistungsbezogene) Homogenisierungsstrukturen auf. Hierauf verweisen jedenfalls weiterhin bestehende Praktiken wie Einschulungszurückstellungen und Klassenwiederholungen (van Ackeren und Kühn 2017), teils überlagert von segregierenden Effekten freier Schulwahl in der Primarstufe (Helbig 2010), sowie auch der vielerorts weiterhin praktizierte Ausschluss aus der Grundschule mittels Überweisung in eine Förderschule. Neben Kindern ohne Aufenthaltsstatus, denen der Zugang zur Schule in Deutschland teilweise verwehrt wird (Funck et al. 2015; Panagiotopoulou und Rosen 2017), besuchen weiterhin 4,2\% aller Kinder einer Jahrgangsstufe auf der Basis sonderpädagogisch verwalteter Diagnostiken eine Förderschule (Kultusministerkonferenz 2020, S. XVII). Das derzeit im Feld der Frühen Bildung diskursbestimmende Präventionsparadigma scheint diese Tendenz noch zu verstärken (kritisch Kelle 2018) und die vorangegangene Reformphase um Transitionsgestaltung und die Flexible Schuleingangsphase (Faust 2006) letztlich zu unterlaufen (Urban et al. 2015), was als deutlicher Hinweis auf verstärkt an Normalität ausgerichteten Topoi und Ansätzen gesehen werden kann (kritisch Diehm 2020). Es ist somit zu fragen, inwieweit die Grundschule ihre vielfach gezeigte pädagogisch-didaktische Innovationskraft weiterführend transformatorisch nutzen kann. 
Dies zeigt sich insbesondere hinsichtlich des Auftrags, sich zu einer inklusiven Grundschule weiterzuentwickeln.

\section{Inklusionsforschung und fachdidaktische Forschung}

Die zunächst als Integrationsforschung bezeichnete Forschungslinie um die wissenschaftlichen Begleitstudien der Modellversuche integrativer Erziehung und Bildung in den 1980er-Jahren entwickelte sich lange Zeit nur fragmentarisch mit Fragen fachdidaktischer Lernprozesse verbunden. Die frühe wissenschaftliche Erschließung der Modellversuche an Grundschulen, die im Anschluss an die erfolgreiche Umsetzung in Kindertageseinrichtungen zunächst in NRW, Bremen, Hessen, Hamburg und dem Saarland auf den Weg gebracht wurden (Schnell 2003), lieferte vor allem ein umfassendes Bild des Reformpotenzials integrativer Erziehung und Bildung für einen professionellen Umgang mit Heterogenität, für Formate des Team-Teachings und für eine kindorientierte Pädagogik und Didaktik insgesamt (zusammenfassend DeppeWolfinger et al. 1990; Eberwein und Knauer 2002) sowie auf einer zweiten Ebene für einen Forschungstypus, der Praxis- und Wissenschaftsanliegen auf der Basis gemeinsam getragener Innovationsbereitschaft produktiv zusammenführte. Lehrpersonen wurden vielfach als (Be)Forscher*innen ihrer eigenen Praxis adressiert (u.a. Valtin 1984) und theoretische Grundlegungen mit konzeptionellen Fragen und konkreten Handlungsanforderungen verknüpft. Die Forschung in dieser frühen Phase war zugleich in Teilen Wirkungsforschung - es wurde unter anderem gezeigt, dass sich bei integrativer Grundschulpraxis keine negativen Effekte auf die Leistungsentwicklung der Kinder in der Breite erkennen lassen und hiermit zugleich für Kinder mit der Zuschreibung eines sonderpädagogischen Förderbedarfs Leistungsvorteile einhergehen (zusammenfassend Müller und Prengel 2013). Ausgehend davon, dass der Leistungsertrag allein nur ein unzureichender Indikator für Gelingen und Schulqualität ist, kann mit Blick auf die Konzeptentwicklung, die pädagogische Qualität und das Potenzial für die Professionalisierung von Lehrpersonen - mithin für das Innovationspotenzial des „Gemeinsamen Lernens“ insgesamt - als originärer Beitrag der Integrationsforschung festgehalten werden, erstmals gezeigt zu haben, dass und wie es möglich ist, alle Kinder ,in der ganzen Bandbreite menschlicher Vielfalt von den Schwerstbehinderten bis hin zu den Hochbegabten gemeinsam zu unterrichten“ (Deppe-Wolfinger et al. 1990, S. 278). Damit widerlegte sie zugleich politisch motivierte Befürchtungen negativer Auswirkungen eines Verzichts auf Stratifizierung.

Auf der Unterrichtsebene wurde hierbei vor allem an bereits entwickelte Formen geöffneten Unterrichts und projektorientierten Lernens angeknüpft (u. a. Hänsel 1986), die in Zusammenarbeit mit den Grundschulen, von denen viele reformpädagogisch arbeiteten, produktiv weiterentwickelt wurden (u. a. Dumke 1991). Die empirische Integrationsforschung im Format der wissenschaftlichen Begleitforschung fokussierte also zunächst vor allem Fragen der pädagogischen Ausgestaltung und der allgemeindidaktischen Konzeption eines an Heterogenität ausgerichteten Unterrichts. Die Fächerstruktur des Grundschulunterrichts nahm jedoch eine untergeordnete Rolle ein und fachbezogene Fragestellungen wurden lediglich im Lichte (fachlicher) Leistungsentwicklung unterschiedlicher Kinder in den „I-Klassen“ un- 
tersucht (Müller und Prengel 2013). Auch im weiteren Verlauf spielten fachdidaktische Fragestellungen in der Integrationsforschung keine prominente Rolle und der Diskurs innerhalb der unterrichtsbezogenen Integrationsforschung rankte sich vielmehr lange auf allgemeindidaktischer Ebene um die Frage des Verhältnisses von Gemeinsamkeit und Individualisierung (Seitz 2006, 2018, 2020).

Dabei wurde von Seiten der bremischen Schulbegleitforschung die Konzeption einer Allgemeinen (integrativen) Didaktik vorgelegt (Feuser 1989), die sich eng an Klafkis Grundlegung Allgemeiner Bildung und der Demokratisierung von Schule orientierte. Von hier aus entwarf Feuser einen fächerübergreifenden, vorhabenorientierten Projektunterricht am ,gemeinsamen Gegenstand“ nach Maßgabe epochaltypischer Schlüsselprobleme. Diese Konzeption bezog zwar am Beispiel der „Gemüsesuppe" fachwissenschaftliche Überlegungen ein, allerdings allein naturwissenschaftliche. Sie begründete sich außerdem entwicklungspsychologisch unter Rekurs auf Piaget und Wygotski, verknüpfte dies aber nicht mit Erkenntnissen zur Domänenspezifität (Sodian 1995) und zur sozialisationsbedingten Kontextualisierung (FöllingAlbers 2005) der kindlichen Wissensentwicklung und der hier anknüpfenden fachdidaktischen Lernvoraussetzungsforschung (Wiesemann und Wille 2014). Folglich wurde hier nicht im engeren Sinne fachdidaktisch argumentiert und die vorgenommene Reduzierung des beispielhaft ausgearbeiteten, fächerübergreifend angelegten Projekts allein auf den physikalischen Prozess ließ vieles aus (Seitz 2005, S. 164 ff.). Insgesamt folgte dieser Ansatz damit einerseits dem Anspruch der Kindzentrierung über Demokratisierung und Projektorientierung und Klafkis bildungstheoretischem, originär in der Grundschulpädagogik entwickelten Konzept, zog aber mit der entwicklungspsychologisch begründeten Tätigkeitsstruktur-, Sachstruktur- und Handlungsstrukturanalyse (Feuser 1995), die eine Verflechtung sachbezogener und kindbezogener Überlegungen in den unterrichtlichen Planungstätigkeiten anzielte, zugleich voraussetzungsvolle und lehrer*innenzentrierte Instrumente in den Unterricht ein, die diesen Anspruch schwächten und sich nicht bruchlos mit fachdidaktischen Begründungen zusammenführen ließen (Seitz 2006).

Demgegenüber stand die aus den Forschungen der Frankfurter Forschergruppe entwickelte Theorie integrativer Prozesse, die ebenso wie Feusers Konzept zunächst für den Elementarbereich entwickelt und dann auf die (Grund-)Schule transferiert wurde. Diese fokussierte zuvorderst die pädagogische Dimension des Lehrer*innenhandelns und rekurrierte auf die Themenzentrierte Interaktion (Cohn 1997), worüber sich eine direkte, aber erst spät für die Inklusionsforschung bearbeitete (vgl. Frohn et al. 2019) Nähe zum Hamburger Modell (Schulz 1980) ergab. Über die hier anknüpfenden Arbeiten Wockens zur Theorie gemeinsamer Lernsituationen (1998) wurden ebenso Bezüge zum Berliner Modell (Heimann et al. 1965) und insgesamt zu einer strukturfunktionalistischen Vorstellung von Unterricht bestärkt. Dieser gesamte Diskurs kam jedoch weitestgehend ohne fachliche Konkretionen oder fachdidaktische Begründungen aus (Seitz 2005).

Fachdidaktische Überlegungen nahmen folglich in der unterrichtsbezogenen Inklusionsforschung insgesamt aus zweierlei Gründen eine Randposition ein: zum einen bedingt durch die Einbettung in reformpädagogisch ausgerichteten Unterricht und die hier entwickelten offenen Unterrichtsformen wie Freiarbeit. Zum anderen 
verstand sich inklusiver Unterricht unter Rekurs auf bildungstheoretische Grundlegungen zuvorderst als ein fächerübergreifender und projektorientierter Unterricht.

Entscheidende Impulse erhielten die integrations- und grundschulpädagogischen Diskurse über die Pädagogik der Vielfalt und die Denkfigur ,egalitärer Differenz“ (vgl. Prengel 1993). In diesem Ansatz wurden Aspekte von Interkulturalität, Geschlecht und Befähigung/Behinderung gedanklich zusammengeführt und als unterschiedliche Dimensionen von Heterogenität auf der Basis der Gleichheit von Rechten und grundlegenden Bedürfnissen konturiert. Dieser bot sowohl für die weitere theoretische Fundierung einer insgesamt an Heterogenität ausgerichteten allgemeinen Didaktik (Klafki 1994) in der Primarstufe wie auch spezifisch der pädagogischen Dimension von Grundschulunterricht (Prengel 1999) zentrale Anknüpfungspunkte. Späterhin wurde er mehr und mehr verwoben mit ungleichheitskritischen Ansätzen der Intersektionalitätsforschung und hiermit verknüpften machtkritischen Forschungslinien um die Ableismus-, Klassismus- und Rassismusforschung und Prozesse des Otherings im Bildungskontext (Riegel 2016; Boger 2019) und von hier aus in theoretische Fundierungen inklusiven Unterrichts integriert (Seitz 2020).

Fachdidaktische Forschungsarbeiten knüpften erst Anfang der 2000er Jahre an die pädagogischen und allgemeindidaktischen Forschungsarbeiten der Integrations-/ Inklusionsforschung an und erschlossen zunächst den Sachunterricht für inklusionspädagogische Fragestellungen (Seitz 2005), der sich vor allem durch den fächerintegrierenden Charakter und die damit gegebene spezifische Nähe zu und Anschlussfähigkeit an bildungstheoretische Grundlegungen der Allgemeinbildung anbot. Ihm kommt neben seiner zweifellos herausgehobenen Stellung als grundschulspezifischer Lernbereich diesbezüglich eine Scharnierfunktion zu, weshalb er im Folgenden genauer beleuchtet wird.

\section{Inklusiver Fachunterricht in der Grundschule - zur Scharnierfunktion des Sachunterrichts}

Der Sachunterricht stellt im Fächerkanon der Grundschule ein relativ junges Fach dar, das in den 1970er-Jahren (in der BRD) das bis dahin dominante Konzept der Heimatkunde ablöste. Er verknüpft ein breites Feld perspektivbezogener wie perspektivenübergreifender Bildungsanteile (GDSU 2013) unter der - mehr oder weniger profilierten - Klammer des Auftrags grundlegender Bildung und einer propädeutischen Komponente und verweist damit auf mehrere fachliche Bezugswissenschaften. Wie sich am Forschungsdiskurs ablesen lässt, hat keine andere Fachdidaktik inklusionsbezogene Fragestellungen so früh aufgegriffen wie der Sachunterricht und er stellt bis heute das diesbezüglich am differenziertesten beforschte und konzeptionell am weitesten ausgearbeitete Feld dar (vgl. Pech und Schomaker 2013, S. 341; Seitz 2018; Simon 2018; Pech et al. 2018).

Den relativ frühen Beginn der fachbezogenen Bearbeitung inklusiven fachlichen Lernens innerhalb der Sachunterrichtsdidaktik markieren im Wesentlichen die empirischen Studien zum inklusiven Sachunterricht von Seitz (2005) und Schomaker (2007), wobei insbesondere die Studie von Seitz in der Inklusionsforschung breit rezipiert wurde. Im diesbezüglichen sachunterrichtsdidaktischen Diskurs wurden die 
Arbeiten jedoch nur partiell aufgenommen und in den sachunterrichtsspezifischen empirischen Designs fanden inklusive Settings weiterhin kaum Berücksichtigung erst im Nachgang der internationalen Verpflichtung zum Aufbau eines inklusiven Schulsystems (United Nations 2006) zeigte sich hier ein Aufschwung (Giest et al. 2011; Pech et al. 2018, 2019). Unter den in diesem Zuge entstandenen Veröffentlichungen befinden sich sowohl grundlegende konzeptionelle Arbeiten (Pech und Schomaker 2013) als auch konkrete Planungshilfen für inklusiven Sachunterricht (vgl. Kahlert und Heimlich 2012; Gebauer und Simon 2012), welche allerdings noch kaum empirisch erschlossen worden sind (vgl. Pech und Rauterberg 2016; Seitz 2018). Dessen ungeachtet scheinen inklusionsbezogene Reflektionen im sachunterrichtsdidaktischen Diskurs insgesamt folglich weiterhin erst in Teilen angekommen zu sein.

Aktuelle sachunterrichtsbezogene Studien thematisieren diesbezüglich ein breites Spektrum mittelbar inklusionsbezogener Fragestellungen und heben auf Ziele und Methoden inklusiven fachlichen Lernens, Prozesse des Otherings sowie auf Fragen der fachbezogenen Professionalisierung ab (Pech et al. 2019). Es finden sich außerdem verschiedene sonderpädagogisch ausgerichtete Beiträge und Arbeiten, die von Differenzsetzungen ausgehen und diese mit einem entsprechend selbstreferentiellen Zugang bearbeiten - etwa indem nach den spezifischen Lernweisen von Kindern „mit“ sonderpädagogischem Förderbedarf im Sachunterricht in Grundschulen gefragt wird und hierzu konzeptionelle Antworten entwickelt werden (Kahlert und Heimlich 2012; Blumberg et al. 2016). Diese Arbeiten fragen somit in einem eher kategorialen Zugang nach Spezifika von - hierüber definierten - Personengruppen, während Prozesse der Herstellung von Normalität und Differenz hier nicht im Fokus der Analysen stehen. Sie sind dabei nicht substantiell verbunden sind mit dem Diskurs der Inklusionsforschung, der sich abgrenzend hiervon primär aus den Erkenntnissen der ungleichheitskritischen Forschungslinien und hier hinterlegten Theorierahmen speist (u.a. Foucault 1978). Hier anknüpfend decken die in diesem Feld entstehenden Arbeiten hegemoniale Praktiken der Herstellung von Normalität und Differenz und deren machtvoller Vermaterialisierung u. a. in Form von Diagnosen „sonderpädagogischen Förderbedarfs“ auf, auf welche die Sonderpädagogik in Forschung und Konzeptbildung referenziert, und kritisieren deren ungleichheitsverstärkende Wirksamkeit (Boger 2019).

Ein sonderpädagogisch informierter Zugang zu fachdidaktischen Fragestellungen, der sich auf der Konzeptebene gut verbinden lässt mit der aktuell in der Sonderpädagogik insgesamt dominierenden Profilierung über diagnostische Instrumente und Technologien der Prävention und Intervention im Rahmen des Classroom-Managements (Hillenbrand und Hennemann 2012), steht somit einerseits unverbunden neben den angesprochenen dekonstruierenden Ansätzen der Inklusionsforschung, zeigt sich jedoch andererseits im Feld der fachdidaktischen Forschungslinien innerhalb einiger Diskursstränge durchaus als anschlussfähig - und zwar vor allem an die Ausprägungen kompetenzorientierter, empirischer Lehr-Lernforschung, die in Deutschland in Folge der PISA-Debatten breiten Aufschwung erfuhren. Hierauf rekurrierende Forschungsprogramme in den Fachdidaktiken fokussieren dabei prominent die Effektivität von Unterricht im Sinne der Leistungssteigerung und des Wissenserwerbs (Klieme und Rakoczy 2008). Sonderpädagogische Deutungsange- 
bote inklusionsdidaktischer Fragestellungen versprechen somit über die immanenten Differenzkonstruktionen Anschlussfähigkeit an diese wissenschaftlichen Diskurse um die Zielidee von Kompetenzsteigerung auf der Unterrichtebene.

Während sich also die Grundschulforschung einerseits als Forschungsfeld durch einen breiten und kritischen Diskurs zur (Bildungs-)Ungleichheit auszeichnet (u.a. Ramseger und Wagener 2008; Skorsetz et al. 2020), findet sich dies andererseits in der fachdidaktischen empirischen Bildungsforschung, in der ein Fokus auf den Fachunterricht in der Sekundarstufe und hier - mehr oder weniger implizit - auf einen Unterricht im stratifizierten System der unterschiedlichen Schulformen dominiert, nicht in der gleichen Form und Intensität wieder. Fragen von Heterogenität werden vielmehr weiterhin vor allem in der grundschulbezogenen fachdidaktischen Forschung bearbeitet. Insbesondere in den letzten fünf Jahren sind hier viele verschiedene Forschungsaktivitäten explizit zum Feld inklusionsbezogener, fachdidaktischer Primarstufendidaktik erkennbar (u.a. Hennies und Ritter 2014; Peter-Koop et al. 2015). Diese Diskurse sind jedoch nur in Teilen verbunden mit ungleichheitsbezogenen Fragestellungen und finden sich bezogen auf die Sekundarstufe nicht in der gleichen Form wieder.

Zugleich durchdringen und beleuchten aktuelle unterrichtsbezogene Forschungsarbeiten in der Grundschul- und Inklusionsforschung ungleichheitsbezogene Fragestellungen in vielfältiger Weise (u.a. Huf und Schnell 2015) und analysieren mit Rassismen und Klassismen unterlegte Praktiken der Herstellung von Differenz (Bräu 2015; Diehm 2020) sowie der intergenerational bedingten Ambivalenzen im Lichte der Heterogenität von Lerngruppen (Prengel und Heinzel 2012). Diese Arbeiten referenzieren vielfach auf Kategorien der Kindheitsforschung und die pädagogische Dimension von Unterricht und Schulleben unter den Bedingungen disparater Lebensbedingungen von Kindern und greifen nur selten explizit fachdidaktische Fragestellungen auf (Kucharz 2015).

Im Sachunterricht, der sich spezifisch dadurch auszeichnet, dass Ungleichheit(en) und differente Lebenswelten auch Inhalt der Fachdidaktik selbst sind, hat sich dabei in besonderer Weise ein breiter(er) Diskurs zur Demokratisierung des Unterrichts und zur Partizipation entwickelt (u.a. Prote 2003; Simon 2018; Baumgardt 2018; Siebach et al. 2019), womit ein direkter Anschluss an inklusionspädagogische Unterrichtskonzeptionen geschaffen ist, denn Partizipation im Sinne von Teilhabe und Mitbestimmung ist hier kennzeichnend (Simon und Pech 2019; Seitz 2020). Der Sachunterricht, der in seiner Grundkonzeption Partizipation als Struktur- und Prozessmerkmal zulässt und als der Ort verstanden werden kann, an dem Fragen und Interessen von Kindern in divergenten Lebenslagen Raum erhalten, bietet sich hier in besonderer Weise an, um Anknüpfungsunkte auch zur Ausdifferenzierung der weiteren grundschulbezogenen Lernbereiche und zu übergreifenden Fragen zu generieren. Die oben angesprochene Scharnierfunktion des Sachunterrichts ist damit weit mehr als nur eine Frage des Übergangs zwischen allgemeindidaktischen Überlegungen und deren fachlich-inhaltlicher Ausdifferenzierung, sondern auch im Hinblick auf pädagogische Hinterlegungen fachdidaktisch reflektierten Unterrichts bedeutsam.

Damit ist ein gedanklicher Übergang geschaffen von fachbezogenen und fachübergreifenden Unterrichtsentwicklungsprozessen zum professionellen Handeln (Helsper 2014), denn die pädagogische Ausgestaltung von Unterricht ist untrennbar 
mit den Lehrpersonen und deren Professionalisierung sowie dem jeweiligen schulischen Wissensdiskurs verbunden (Seitz 2020). Die komplexen Dynamiken, die dem folgend mit inklusionsbezogenen Anforderungen auf der Ebene von Schulen als lernenden Organisationen (Berkemeyer et al. 2015) einhergehen, lassen sich in der Trias von inklusiven Strukturen, Kulturen und Praktiken bearbeiten (Booth und Ainscow 2017; Boban und Hinz 2015) und machen die Potenziale von Forschungsprogrammen deutlich, die fachdidaktisch reflektierte, inklusionsbezogene Forschungsfragen mit Fragen der inklusiven Schulentwicklung und (Fach-)Unterrichtsentwicklung im Ganzen und dem hier eingebundenen professionellen Handeln verbinden.

\section{Implikationen und Perspektiven}

Die Zusammenstellungen und Überlegungen machen deutlich, dass der Diskurs zur fachlichen inklusiven Bildung - obgleich er weiter fortgeschritten ist als im Bereich der Sekundarstufe - auch in der Primarstufe noch in den Anfängen steckt, aber viel Potenzial bietet.

Bezogen auf das professionelle Handeln von Lehrpersonen im inklusiven Unterricht wurde von Seiten der Inklusionsforschung als spezifische handlungsleitende Orientierung herausgearbeitet, Gemeinsamkeit und Individualisierung nicht als gegeneinander abzuwägende Aspekte der Unterrichtsplanung zu bearbeiten, sondern gleichzeitig in einer Unterrichtssituation hervorzubringen und didaktisch abzusichern (Scheidt 2017), was auf das Professionalisierungspotenzial inklusiver Unterrichtspraxis selbst verweist und einen substantiellen Ausgangspunkt für weitere fachdidaktisch angelegte, inklusionsbezogene Unterrichtsforschung bietet (Seitz 2020).

Dabei wäre es weiterführend erkenntnisreich, auch die „Anlage des Faches“(Pech und Schomaker 2013) aus inklusionspädagogischer Perspektive einer kritischen Reflexion zuzuführen und beispielsweise zu erörtern, inwiefern den oben angestellten bildungstheoretischen Grundlagen inklusiver Bildung im jeweiligen Fach nachgekommen wird bzw. werden kann und inwiefern sich aus einer Orientierung an dieser Veränderungsimpulse ergeben. Auch ist eine Intensivierung fachbezogener Forschungen zu sozialer und fachlicher Partizipation angezeigt. Empirisch fundierte Reflektionen der „Kontur“ der Fachdidaktiken als Disziplinen und ihrer spezifischen Bedingungen für curriculare Partizipation könnten so gewinnbringend mit empirischen Analysen disziplinärer Bestände hinsichtlich ihres Beitrages zu Prozessen der Herstellung von Differenz im und durch Fachunterricht verbunden werden.

Die Darlegungen zeigen damit insgesamt, dass für eine substantielle Weiterentwicklung vor allem komplexe Forschungsprogramme impliziert sind, die (inklusions)pädagogische Fragestellungen mit fachlichen Fragen verknüpfen, dabei aber die ungleichheitsbezogenen Ambivalenzen der Grundschule als Institution nicht ausblenden, sondern mit reflektieren und hierfür das professionelle Handeln in den Blick nehmen.

Vielversprechend scheinen daher zum einen insbesondere Arbeiten, die auch $\mathrm{Zu}$ sammenhänge von Schulentwicklung und Professionalisierung aufnehmen und so 
die unterschiedlichen Ebenen der Entwicklung inklusiver Grundschulen im Modus diskursiver Aushandlungsprozesse vom ,Sollen zum Wollen“ (Schratz 2015) fokussieren. Denn inklusive Grundschulen sind im deutschen Bildungssystem in widerspruchsreiche Politiken und Steuerungsprozesse eingebunden, die über entsprechende Rekontextualisierungen in den schulkulturellen Diskursen der Einzelschulen bearbeitet werden und hier bedeutsam sind für die handlungsleitenden Orientierungen der Lehrpersonen (Helsper 2014) auch auf fachlicher Ebene. Inklusive Handlungspraxis in Grundschulen ist somit wie bereits angedeutet weder eine Frage der „Haltung“, die sich der Lernbarkeit in Teilen entziehen würde, noch lässt sie sich auf den Erwerb fachspezifischer Wissensanteile und Kompetenzen (angehender) Lehrpersonen reduzieren. Beides würde zu kurz greifen, denn professionelles Handlungswissen zeichnet sich spezifisch durch die Zusammenführung unterschiedlicher Wissensformen aus, die zugleich berufsbiografisch hinterlegt sind und schulisch geprägte Orientierungsrahmen aufweisen, was einen breiteren gedanklichen Rahmen impliziert, der Erkenntnisse der unterschiedlichen Professionalisierungsansätze verbindet (Kaiser et al. 2020).

Hiermit angesprochene Forschungen zur fachbezogenen inklusionsorientierten Grundschullehrer*innenbildung stecken allerdings noch in den Anfängen (Simon 2018). Insbesondere mit Blick auf Praxisanteile im Studium zeigen sich diesbezüglich bedeutsame Ambivalenzen, denn entsprechende Erfahrungen verhalten sich bedingt durch politisch motivierte Restriktionen inklusiver Bildungspraxis in Grundschulen vielfach nicht bruchlos zu den universitär vermittelten Konzeptionen und professionellen Anforderungen, was für Studierende verschiedene Friktionen mit sich bringt, die einer reflexiven Bearbeitung bedürfen (Seitz 2018; Lau et al. 2019). Impliziert sind daher vor allem kasuistische Formate und bewusst gesetzte Irritationen, um paradoxe Anforderungen nicht als lähmend zu erleben, sondern handlungsfähig zu bleiben und Gestaltungsspielräume für Weiterentwicklung und Professionalisierung zu generieren und zu nutzen (Seitz und Slodczyk 2020).

Damit wäre zugleich ein Anschluss an Basiskonzepte der Grundschule und ihr Selbstverständnis als Ort der demokratisch verankerten Grundbildung und die hieran gebundene Rolle von Lehrpersonen und Wissenschaftler*innen gegeben. Denn wie beispielhaft bereits in den Marburger Grundschulprojekten Klafkis gezeigt, ist der Rolle von Lehrpersonen politische Verantwortlichkeit und Handlungsfähigkeit inhärent, was für Klafki impliziert, nicht allein innerhalb institutioneller Rahmenbedingungen $\mathrm{zu}$ agieren, sondern erkannte Veränderungsmöglichkeiten hin $\mathrm{zu}$ humaneren und demokratischeren Bildungsstrukturen aktiv zu entwickeln und umzusetzen (Klafki et al. 1982; Braun et al. 2018). Und hierzu benötigen Lehrpersonen eine gleichermaßen verantwortlich agierende Wissenschaft.

Open Access Dieser Artikel wird unter der Creative Commons Namensnennung 4.0 International Lizenz veröffentlicht, welche die Nutzung, Vervielfältigung, Bearbeitung, Verbreitung und Wiedergabe in jeglichem Medium und Format erlaubt, sofern Sie den/die ursprünglichen Autor(en) und die Quelle ordnungsgemäß nennen, einen Link zur Creative Commons Lizenz beifügen und angeben, ob Änderungen vorgenommen wurden.

Die in diesem Artikel enthaltenen Bilder und sonstiges Drittmaterial unterliegen ebenfalls der genannten Creative Commons Lizenz, sofern sich aus der Abbildungslegende nichts anderes ergibt. Sofern das betreffende Material nicht unter der genannten Creative Commons Lizenz steht und die betreffende Handlung 
nicht nach gesetzlichen Vorschriften erlaubt ist, ist für die oben aufgeführten Weiterverwendungen des Materials die Einwilligung des jeweiligen Rechteinhabers einzuholen.

Weitere Details zur Lizenz entnehmen Sie bitte der Lizenzinformation auf http://creativecommons.org/ licenses/by/4.0/deed.de.

\section{Literatur}

van Ackeren, I., \& Kühn, S. M. (2017). Homogenität und Heterogenität im deutschen Schulsystem. In T. Bohl, J. Budde \& M. Rieger-Ladich (Hrsg.), Umgang mit Heterogenität in Schule und Unterricht (S. 175-190). Bad Heilbrunn: Klinkhardt UTB.

Arnold, K.-H., Hauenschildt, K., Schmidt, B., \& Ziegenmeyer, B. (Hrsg.). (2010). Zwischen Fachdidaktik und Stufendidaktik. Perspektiven für die Grundschulpädagogik. Wiesbaden: Springer.

Baumgardt, I. (2018). Partizipation im inklusiven Sachunterricht - ein Beitrag für die demokratische Schulund Unterrichtsentwicklung? In D. Pech, C. Schomaker \& T. Simon (Hrsg.), Sachunterrichtsdidaktik \& Inklusion. Ein Beitrag zur Entwicklung (S. 26-38). Baltmannsweiler: Schneider.

Berkemeyer, J., Berkemeyer, N., \& Meetz, F. (Hrsg.). (2015). Professionalisierung und Schulleitungshandeln. Wege und Strategien der Personalentwicklung an Schulen. Weinheim, Basel: Beltz Juventa.

Blumberg, E., Hellmich, F., \& Fromme, T. (2016). Entwicklung naturwissenschaftlich-technischer LehrLernangebote für inklusiven Sachunterricht. In K. Liebers, B. Landwehr, A. Marquardt \& K. Schlotter (Hrsg.), Lernprozessbegleitung und adaptives Lernen in der Grundschule - Forschungsbezogene Beiträge (S. 235-236). Wiesbaden: Springer VS.

Boban, I., \& Hinz, A. (Hrsg.). (2015). Erfahrungen mit dem Index für Inklusion. Kindertageseinrichtungen und Grundschulen auf dem Weg. Bad Heilbrunn: Klinkhardt.

Boger, M.-A. (2019). Theorien der Inklusion. Die Theorie der trilemmatischen Inklusion zum Mitdenken. Münster: edition assemblage.

Booth, T., \& Ainscow, M. (2017). Index für Inklusion. Weinheim, Basel: Beltz.

Bräu, K. (2015). Soziale Konstruktionen in Schule und Unterricht - eine Einführung. In K. Bräu \& C. Schlickum (Hrsg.), Soziale Konstruktionen in Schule und Unterricht. Zu den Kategorien Leistung, Migration, Geschlecht, Behinderung, Soziale Herkunft und deren Interdependenzen (S. 17-32). Opladen, Berlin: Budrich.

Braun, K.-H., Stübig, F., \& Stübig, H. (Hrsg.). (2018). Erziehungswissenschaftliche Reflexion und pädagogisch-politsches Engagement. Wolfgang Klafki weiterdenken. Wiesbaden: Springer VS.

Cohn, R. C. (1997). Von der Psychoanalyse zur themenzentrierten Interaktion (13. Aufl.). Stuttgart: KlettCotta.

Deppe-Wolfinger, H., Prengel, A., \& Reiser, H. (1990). Integrative Pädagogik in der Grundschule. Bilanz und Perspektiven der Integration behinderter Kinder in der Bundesrepublik Deutschland 1976-1988. Weinheim: Juventa.

Diehm, I. (2020). Differenz - die pädagogische Herausforderung in der Schule für alle Kinder. In N. Skorsetz, M. Bonanati \& D. Kucharz (Hrsg.), Diversität und soziale Ungleichheit - Herausforderungen an die Integrationsleistung der Grundschule (S. 9-19). Frankfurt a.M: Springer VS.

Dumke, D. (Hrsg.). (1991). Integrativer Unterricht. Gemeinsames Lernen von Behinderten und Nichtbehinderten. Weinheim: Deutscher Studien Verlag.

Eberwein, H. \& Knauer, S. (Hrsg.). (2002). Handbuch Integrationspädagogik. Weinheim \& Basel: Beltz.

Einsiedler, W. (2005). Grundlegende Bildung. In W. Einsiedler, M. Götz, H. Hacker, J. Kahlert, R. Keck \& U. Sandfuchs (Hrsg.), Handbuch Grundschulpädagogik und Grundschuldidaktik (2. Aufl. S. 217-227). Bad Heilbrunn: Klinkhardt.

Faust, G. (2006). Zum Stand der Einschulung und der neuen Schuleingangsstufe in Deutschland. Zeitschrift für Erziehungswissenschaft, 9(3), 328-347.

Faust-Siehl, G., Garlichs, A., Ramseger, J., Schwarz, H., \& Warm, U. (1996). Die Zukunft beginnt in der Grundschule. Empfehlungen zur Neugestaltung der Primarstufe. Reinbek: Rowohlt.

Feuser, G. (1989). Allgemeine integrative Pädagogik und entwicklungslogische Didaktik. Behindertenpädagogik, 28(1), 4-48.

Feuser, G. (1995). Behinderte Kinder und Jugendliche. Zwischen Integration und Aussonderung. Darmstadt: Wissenschaftliche Buchgesellschaft.

Fölling-Albers, M. (2005). Soziokulturelle Bedingungen der Kindheit. In W. Einsiedler, M. Götz, A. Hartinger, F. Heinzel \& J. Kahlert (Hrsg.), Handbuch Grundschulpädagogik und-didaktik (S. 155-166). Bad Heilbrunn: Klinkhardt. 
Foucault, M. (1978). Dispositive der Macht. Michel Foucault über Sexualität, Wissen und Wahrheit. Berlin: Merve.

Frohn, J., Brodesser, E., Moser, V., \& Pech, D. (2019). Einführung. In dies (Hrsg.), Inklusives Lehren und Lernen. Allgemein- und fachdidaktische Grundlagen (S. 9-20). Bad Heilbrunn: Klinkhardt.

Funck, B.J., Karakaşoğlu, Y., \& Vogel, D. (2015). „Es darf nicht an Papieren scheitern“. Theorie und Praxis der Einschulung von papierlosen Kindern in Grundschulen. Frankfurt a. M.: GEW.

Gebauer, M., \& Simon, T. (2012). Inklusiver Sachunterricht konkret: Chancen, Grenzen, Perspektiven. www.widerstreit-sachunterricht.de, Nr. 18, Oktober 2012. http://www.widerstreit-sachunterricht.de/ ebeneI/superworte/inklusion/gebauer_simon.pdf. Zugegriffen: 2. Juni 2020.

Gesellschaft für Didaktik des Sachunterrichts (GDSU) (Hrsg.). (2013). Perspektivrahmen Sachunterricht. Bad Heilbrunn: Klinkhardt.

Giest, H., Kaiser, A., \& Schomaker, C. (Hrsg.). (2011). Sachunterricht-auf dem Weg zur Inklusion. Bad Heilbrunn: Klinkhardt.

Hänsel, D. (Hrsg.). (1986). Das Projektbuch Grundschule. Weinheim: Beltz.

Heimann, P., Otto, G., \& Schulz, W. (Hrsg.). (1965). Unterricht. Analyse und Planung. Hannover: Schroedel.

Helbig, M. (2010). Neighbourhood does matter! Soziostrukturelle Nachbarschaftscharakteristika und Bildungserfolg. Kölner Zeitschrift für Soziologie und Sozialpsychologie, 62(4), 655-679.

Helsper, W. (2014). Lehrerprofessionalität - der strukturtheoretische Professionsansatz zum Lehrberuf. In E. Terhart, H. Bennewitz \& M. Rothland (Hrsg.), Handbuch der Forschung zum Lehrerberuf (2. Aufl. S. 216-240). Münster: Waxmann.

Hennies, J., \& Ritter, M. (Hrsg.). (2014). Deutschunterricht in der Inklusion. Auf dem Weg zu einer inklusiven Deutschdidaktik. Stuttgart: Fillibach.

Hillenbrand, C., \& Hennemann, T. (2012). Unterrichtsstörungen vermeiden - durch gutes Classroom Management. Nach elf Prinzipien Unterricht strukturieren. Schulverwaltung: Zeitschrift Für Schulverwaltung und Schulmanagement. Nordrhein-Westfalen, 23(4), 109-111.

Huf, C., \& Schnell, I. (Hrsg.). (2015). Inklusive Bildung in Kita und Grundschule. Stuttgart: Kohlhammer.

Kahlert, J., \& Heimlich, U. (2012). Inklusionsdidaktische Netze - Konturen eines Unterrichts für alle (dargestellt am Beispiel des Sachunterrichts). In U. Heimlich \& J. Kahlert (Hrsg.), Inklusion in Schule und Unterricht. Wege zur Bildung für alle (S. 153-190). Stuttgart: Kohlhammer.

Kaiser, A., \& Pech, D. (Hrsg.). (2004). Integrative Dimensionen des Sachunterrichts. Neuere Zugangsweisen. Baltmannsweiler: Schneider.

Kaiser, M., Seitz, S., \& Slodczyk, N. (2020). Expertise als übergreifendes Paradigma der Professionalisierungsforschung zur inklusionsbezogenen Fortbildung von Lehrpersonen. Qualifizierung für Inklusion 2 (1). https://www.qfi-oz.de/index.php/inklusion/article/view/30. Zugegriffen: 15. Aug. 2020.

Kelle, H. (2018). Entgrenzung der vorschulischen Diagnostik. Zeitschrift für Grundschulforschung, 11(1), 85-100.

Klafki, W. (1994). „Recht auf Gleichheit - Recht auf Differenz“ in bildungstheoretischer Perspektive. Neue Sammlung, 34(4), 579-594.

Klafki, W., Scheffer, U., Koch-Priewe, B., Stöcker, H., Huschke, P., \& Stang, H. (Hrsg.). (1982). Schulnahe Curriculumentwicklung und Handlungsforschung. Forschungsbericht des Marburger Grundschulprojektes. Weinheim, Basel: Beltz.

Klieme, E., \& Rakoczy, K. (2008). Empirische Unterrichtsforschung und Fachdidaktik. Outcome-orientierte Messung und Prozessqualität des Unterrichts. Zeitschrift für Pädagogik, 54(2), 222-237.

Kucharz, D. (2015). Inklusiver Sachunterricht. In C. Huf \& I. Schnell (Hrsg.), Inklusive Bildung in Kita und Grundschule (S. 221-236). Stuttgart: Kohlhammer.

Kultusministerkonferenz (KMK) (2020). Sonderpädagogische Förderung in Schulen 2009 bis 2018. https://www.kmk.org/fileadmin/Dateien/pdf/Statistik/Dokumentationen/Dok223_SoPae_2018.pdf. Zugegriffen: 2. Juni 2020.

Lau, R., Heinrich, M., \& Lübeck, A. (2019). Professionalisierung in Spannungsfeldern von Inklusion durch Fortbildung. In M. Heinrich \& G. Klewin (Hrsg.), Praxisforschung und Transfer. WE_OS Jahrbuch 2019 (S. 82-99). https://www.biejournals.de/index.php/we_os/issue/view/263/127. Zugegriffen: 02. Juni 2020.

Miller, S., Martschinke, S., Götz, M., Hartinger, A., Kucharz, D., Liebers, K., \& Möller, K. (2019). Diskussion des Selbstverständnisses der Grundschulpädagogik als Disziplin. In C. Donie, F. Foerster, M. Obermayr, A. Deckwerth, G. Kammermeyer, G. Lenske, M. Leuchter \& A. Wildemann (Hrsg.), Grundschulpädagogik zwischen Wissenschaft und Transfer (S. 22-33). Wiesbaden: Springer VS.

Müller, F. J., \& Prengel, A. (2013). Empirische Zugänge zu Inklusion in der Früh- und Grundschulpädagogik. Zeitschrift für Grundschulforschung, 7(1), 7-20. 
Panagiotopoulou, A., \& Rosen, L. (2017). Zur Inklusion von geflüchteten Kindern und Jugendlichen in das deutsche Schulsystem. http://www.bpb.de/gesellschaft/migration/kurzdossiers/258059/inklusion-indas-schulsystem. Zugegriffen: 2. Juni 2020.

Pech, D., \& Rauterberg, M. (2016). Wozu Didaktik? Ein Beitrag zum Verhältnis von Sachunterrichtsdidaktik und Inklusion. In J. Riegert \& O. Musenberg (Hrsg.), Didaktik und Differenz (S. 134-147). Bad Heilbrunn: Klinkhardt.

Pech, D., \& Schomaker, C. (2013). Inklusion und Sachunterrichtsdidaktik. Stand und Perspektiven. In K.-E. Ackermann, O. Musenberg \& J. Riegert (Hrsg.), Geistigbehindertenpädagogik!? Disziplin Profession - Inklusion (S. 341-359). Oberhausen: Athena.

Pech, D., Schomaker, C., \& Simon, T. (Hrsg.). (2018). Sachunterrichtsdidaktik \& Inklusion. Ein Beitrag zur Entwicklung. Baltmannsweiler: Schneider.

Pech, D., Schomaker, C., \& Simon, T. (Hrsg.). (2019). Inklusion im Sachunterricht. Perspektiven der Forschung. Bad Heilbrunn: Klinkhardt.

Peschel, F. (2006a). Offener Unterricht. Idee, Realität, Perspektive und ein praxiserprobtes Konzept zur Diskussion 1. Allgemeindidaktische Überlegungen. Baltmannsweiler: Schneider.

Peschel, F. (2006b). Offener Unterricht. Idee, Realität, Perspektive und ein praxiserprobtes Konzept zur Diskussion 2. Fachdidaktische Überlegungen. Baltmannsweiler: Schneider.

Peter-Koop, A., Rottmann, T., \& Lüken, M. (2015). Inklusiver Mathematikunterricht in der Grundschule. Oldenburg: Mildenberger.

Prengel, A. (1993). Pädagogik der Vielfalt. Verschiedenheit und Gleichberechtigung in Interkultureller, Feministischer und Integrativer Pädagogik. Opladen: Leske + Budrich.

Prengel, A. (1999). Vielfalt durch gute Ordnung im Anfangsunterricht. Opladen: Leske + Budrich.

Prengel, A., \& Heinzel, F. (2012). Heterogenität als Grundbegriff inklusiver Pädagogik. Zeitschrift Für Inklusion, (3). https://www.inklusion-online.net/index.php/inklusion-online/article/view/39. Zugegriffen: 2. Juni 2020.

Prote, I. (2003). Partizipation als Schlüsselqualifikation für das Demokratie-Lernen in der Grundschule. In H.-W. Kuhn (Hrsg.), Sozialwissenschaftlicher Sachunterricht. Konzepte, Forschungsfelder, Methoden (S. 39-51). Herbolzheim: Centaurus.

Ramseger, J., \& Wagener, M. (Hrsg.). (2008). Chancenungleichheit in der Grundschule. Ursachen und Wege aus der Krise. Wiesbaden: VS.

Richter, D. (2002). Sachunterricht - Ziele und Inhalte. Baltmannsweiler: Schneider.

Riegel, C. (2016). Bildung - Intersektionalität - Othering. Bielefeld: transcript.

Scheidt, K. (2017). Inklusion. Im Spannungsfeld von Individualisierung und Gemeinsamkeit. Baltmannsweiler: Schneider.

Schnell, I. (2003). Geschichte schulischer Integration. Gemeinsames Lernen von SchülerInnen mit und ohne Behinderung in der BRD seit 1970. Weinheim: Juventa.

Schomaker, C. (2007). Der Faszination begegnen. Ästhetische Zugangsweisen im Sachunterricht für alle Kinder. Oldenburg: Carl von Ossietzky Universität, Didaktisches Zentrum.

Schratz, M. (2015). Personalentwicklung braucht Leadership. In J. Berkemeyer, N. Berkemeyer \& F. Meetz (Hrsg.), Professionalisierung und Schulleitungshandeln. Wege und Strategien der Personalentwicklung an Schulen (S. 70-95). Weinheim: Beltz.

Schulz, W. (1980). Unterrichtsplanung. München: Urban \& Schwarzenberg.

Seitz, S. (2005). Zeit für inklusiven Sachunterricht. Baltmannsweiler: Schneider.

Seitz, S. (2006). Inklusive Didaktik: Die Frage nach dem ,Kern der Sache‘. Zeitschrift für Inklusion 1 (1). http://inklusiononline.net/index.php/inklusion/article/view/15/15. Zugegriffen: 15. Aug. 2020.

Seitz, S. (2014). Inklusion in der Grundschule. In E.-K. Franz, S. Trumpa \& I. Esslinger-Hinz (Hrsg.), Inklusion. Eine Herausforderung für die Grundschulpädagogik (S. 24-32). Baltmannsweiler: Schneider.

Seitz, S. (2018). Forschung zu inklusivem Sachunterricht - Bestandsaufnahme und Perspektiven. In D. Pech, C. Schomaker \& T. Simon (Hrsg.), Sachunterrichtsdidaktik \& Inklusion. Ein Beitrag zur Entwicklung (S. 96-111). Baltmannsweiler: Schneider.

Seitz, S. (2020). Dimensionen inklusiver Didaktik - Personalität, Sozialität und Komplexität. Zeitschrift für Inklusion. Vol. 15, No. 2. https://www.inklusion-online.net/index.php/inklusion-online/article/view/ 570. Zugegriffen: 15. Aug. 2020.

Seitz, S., \& Slodczyk, N. (2020). Fortbildung der Fortbildner*innen. In R. Schneider-Reisinger \& M. Oberlechner (Hrsg.), Diversitätssensible PädagogInnenbildung in Forschung und Praxis: Utopien, Ansprüche und Herausforderungen (S. 118-127). Opladen: Budrich.

Siebach, M., Simon, J., \& Simon, T. (Hrsg.). (2019). Ich und Welt verknüpfen. Allgemeinbildung, Vielperspektivität, Partizipation und Inklusion im Sachunterricht. Baltmannsweiler: Schneider. 
Simon, T. (2018). Beiträge zur Entwicklung einer inklusionsorientierten Sachunterrichtsdidaktik unter besonderer Berücksichtigung von Fragen der Unterrichtsplanung, diagnostischen Handelns und Fragen der Schüler*innenpartizipation. Paderborn: UPB.

Simon, T. (2020). Die Grundschule als Ort der Demokratie? Für alle? In N. Böhme, B. Dreer, H. Hahn, S. Heinecke, G. Mannhaupt \& S. Tänzer (Hrsg.), Mythen, Widersprüche und Gewissheiten der Grundschulforschung. Eine wissenschaftliche Bestandsaufnahme nach 100 Jahren Grundschule. Wiesbaden: Springer VS.

Simon, T. \& Pech, D. (2019). Partizipation. In J. Frohn, E. Brodesser, V. Moser \& D. Pech (Hrsg.), Inklusives Lehren und Lernen. Allgemein- und fachdidaktische Grundlagen (S. 40-42). Bad Heilbrunn: Klinkhardt.

Skorsetz, N., Bonanati, M., \& Kucharz, D. (Hrsg.). (2020). Diversität und soziale Ungleichheit - Herausforderungen an die Integrationsleistung der Grundschule. Wiesbaden: Springer VS.

Sodian, B. (1995). Entwicklung bereichsspezifischen Wissens. In R. Oerter \& L. Montada. (Hrsg.) Entwicklungspsychologie (S. 622-653). Weinheim, Basel: Beltz.

United Nations (2006). Conventions on the rights of persons with disabilities. http://www.un.org/ disabilities/convention/conventionfull.shtml. Zugegriffen: 2. Juni 2020.

Urban, M., Schulz, M., Meser, K., \& Thoms, S. (Hrsg.). (2015). Inklusion und Übergang. Perspektiven der Vernetzung von Kindertageseinrichtungen und Grundschulen. Bad Heilbrunn: Klinkhardt.

Valtin, R. (1984). Gemeinsam Leben - gemeinsam lernen. Behinderte Kinder in der Grundschule. Konzepte und Erfahrungen. Frankfurt a.M.: Arbeitskreis Grundschule.

Wiesemann, J., \& Wille, F. (2014). Formate didaktischer Forschung zum Sachunterricht. www.widerstreitsachunterricht.de, Nr. 20. http://www.widerstreit-sachunterricht.de/ebeneI/superworte/forschung/ wiesemann_wille.pdf. Zugegriffen: 15. Aug. 2020.

Wocken, H. (1998). Gemeinsame Lernsituationen. Eine Skizze zur Theorie des gemeinsamen Unterrichts. In A. Hildeschmidt \& I. Schnell (Hrsg.), Integrationspädagogik. Auf dem Weg zu einer Schule für alle (S. 37-52). Weinheim: Juventa. 